\title{
ESTRUCTURA FACTORIAL DE LA ESCALA DE ANSIEDAD SOCIAL PARA NIÑOS-REVISADA (SASC-R)
}

\author{
BONIFACIO SANDÍN, PALOMA CHOROT, ROSA M. VALIENTE, \\ MIGUEL A. SANTED Y CARMEN SÁNCHEZ-ARRIBAS ${ }^{1}$ \\ Universidad Nacional de Educación a Distancia, Madrid \\ ${ }^{1}$ Hospital Úbeda, Equipo Salud Mental Villacarrillo (Jaén)
}

(Recibido 17 de noviembre de 1998)

\begin{abstract}
En el presente artículo presentamos los resultados de una investigación sobre la ansiedad social con una muestra de niños y adolescentes no clínicos (rango de edad entre 7 y 16 años). En la investigación estudiamos la estructura factorial de la versión española de la Anxiety Scale for Children-Revised (SASC-R; La Greca y Stone, 1993). Los resultados confirman una estructura tridimensional de la SASC-R (miedo a la evaluación negativa de los iguales, evitación/distress social sobre situaciones generalizadas, y evitación/distress social relativa a situaciones con nuevos compañeros). El análisis factorial de la escala SASC (versión inicial de la escala) dio como resultado dos factores (i.e., miedo a la evaluación negativa de los iguales, y evitación/distress social). Los resultados apoyan los datos referidos en Estados Unidos y porporcionan evidencia transcultural sobre la estructura de la ansiedad social infantojuvenil. Se comentan algunos aspectos relacionados con la utilidad de esta escala.
\end{abstract}

Palabras clave: SASC-R, SASC, ansiedad social, estructura factorial, niños, adolescentes.

\section{Factor structure of the Social Anxiety Scale for Children-Revised}

The present article reports the findings of an investigation on social anxiety with a nonclinical sample of children and adolescents (ranging in age from 7 to 16 years). The study examined the factor structure of the Spanish Anxiety Scale for Children-Revised (SASC-R; La Greca y Stone, 1993). Results supported a three-factor structure of the SASC-R (i.e., fear of negative evaluation from peers, generalized social avoidance and distress, and social avoidance and diestress specific to new peers). Factor analysis of the SASC (the former version of the scale) yielded two factors (i.e., fear of negative evaluation from peers, and social avoidance and distress). Findings support previous U.S. data and provide cross-cultural evidence for the structure of social anxiety in children and adolescents. Some potential utility of the questionnaire is addressed.

Key Words: SASC-R, SASC, social anxiety, factor structure, children, adolescents.

\section{INTRODUCCIÓN}

La fobia o ansiedad social ha pasado de ser "el trastorno de ansiedad abandonado», tal y como ha sido considerado, a ser uno de los trastornos de ansiedad de mayor interés actual. Sin embargo, a pesar del elevado interés general por la investigación de la fobia social, este trastorno permanece virtualmente sin estu-

Correspondencia: Bonifacio Sendín, Facultad de Psicología, Universidad Nacional de Educación a Distancia (UNED), Ciudad Universitaria s/n, 28040 Madrid. diar en los niños y adolescentes (Sandín, 1997, p. 157).

La frecuencia de los miedos y la ansiedad de tipo social durante fases relativamente tempranas del desarrollo constituye un problema de gran interés clínico, educativo y social (Méndez, 1999; Pelechano, 1981; Sandín y Chorot, 1998; Sandín, Chorot, Valiente y Santed, 1998). La elevada incidencia de miedo/ansiedad social durante las edades de la infancia y adolescencia representa un problema crítico, ya que muchos de los trastornos de ansiedad, además de las propias fobias 
sociales, se inician durante la adolescencia. Más aun, los problemas de ansiedad social que transcurren durante la infancia y adolescencia tienden a constituir un factor de riesgo para el desarrollo de otros problemas y trastornos psicopatológicos (depresión, trastornos de ajuste, etc.), además de los trastornos de ansiedad propiamente dichos (para una análisis más amplio, véase Sandín, 1997).

$\mathrm{La}$ ansiedad social interfiere significativamente en las relaciones entre iguales que llevan a cabo los niños y adolescentes, por lo que también puede perturbar y dañar seriamente el desarrollo social de los mismos. Este fenómeno es importante sobre todo durante las fases infantojuveniles, ya que estas etapas constituyen una edad crítica para la socialización y el desarrollo de las relaciones y habilidades interpersonales. La afectación del miedo/ansiedad social puede alcanzar, por tanto, no sólo las fronteras psicopatológicas y clínicas, sino también, y de forma muy especial, los ámbitos educativos, afectando a fenómenos como el desarrollo social, el rechazo entre compañeros, las situaciones que implican evaluación social (p.ej., ansiedad a los exámenes), etc.

A pesar de la especial relevancia clínica que posee la ansiedad social, apenas existen procedimientos psicométricos apropiados para su evaluación durante la infancia y la adolescencia. Los procedimientos psicométricos de autoinforme son medidas particularmente útiles para evaluar la ansiedad social, y pueden ser empleados igualmente para las edades infantiles y juveniles. Muchos de los cuestionarios empleados para estas edades miden la experiencia subjetiva de la ansiedad general, más que de la ansiedad específicamente social (véase Sandín, 1997).

La Social Anxiety Scale for Children (SASC) es un cuestionario breve desarrollado por La Greca, Dandes, Wick,
Shaw y Stone (1988) para evaluar específicamente la ansiedad social en niños y adolescentes. Para la construcción del cuestionario, La Greca et al. (1988) se basaron conceptualmente en las dimensiones sobre ansiedad social obtenidas con adultos por Watson y Friend (1969), esto es, en las dimensiones de miedo a la evaluación negativa (FNE, fear of negative evaluation) y evitación y distress sociales (SAD, social avoidance and distress). La distinción conceptual entre los aspectos subjetivos (miedo a la evaluación negativa) y conductuales (evitación social), presente en el trabajo de Watson y Friend (1969), y enfatizado por la separación que hace Leary (1983) entre "experiencia subjetiva" y "consecuencias conductuales» de la ansiedad social, constituyó el hilo conductor seguido por La Greca et al. (1988) para la construcción del SASC. Esta distinción es importante, ya que algunas personas con ansiedad social subjetiva pueden actuar adecuadamente en los contextos sociales, mientras que otras experimentan distress elevado (experiencia subjetiva de malestar) y evitación social (La Greca y Stone, 1993).

Los estudios factoriales sobre la SASC han demostrado la presencia de ambos factores o dimensiones: (1) El factor FNE reflejaba la experiencia subjetiva de preocupaciones y miedos infantojuveniles relativos a la evaluación efectuada por sus iguales (compañeros, etc.); (2) así mismo, el factor SAD incluía las variables relacionadas con inhibiciones sociales de los niños o evitación de los iguales (evitación social) y experiencias de distress o malestar en situaciones sociales infantojuveniles (distress social) (La Greca et al., 1988). Los autores demostraron que ambas subescalas o dimensiones (FNE y SAD) de la SASC poseen suficiente grado de consistencia interna y de fiabilidad test-retest con muestras de niños de diferentes edades. 
Un posible problema de la SASC es que la subescala SAD no evalúa adecuadamente la evitación social y distress generalizados (los items de esta subescala miden mayoritariamente la evitación social y distress referidos a compañeros desconocidos o poco familiares). Esta fue la razón principal por la cual La Greca y Stone (1993) llevaron a cabo una revisión de la SASC (Social Anxiety Scale for Children-Revised, SASC-R), incorporando nuevos items referidos a evitación social y distress generalizados, bajo la convicción de que tal distinción (situaciones nuevas vs. generalizadas) reviste importantes connotaciones clínicas, tanto de orden teórico como práctico. En este sentido, la distinción entre fobia social específica y fobia social generalizada se sabe que posee una indudable implicación psicopatológica (APA, 1994; Botella y Gallardo, 1999; Echeburúa, 1993; Heimber, Holt, Schneider, Spitzer y Liebowitz, 1993; Sandín, 1997; Sandín y Chorot, 1995).

Tras la adición de nuevos elementos sobre evitación/distress social generalizado, la forma revisada de la escala (i.e., la SASC-R) quedó constituida en 18 items. Los análisis factoriales efectuados por La Greca y Stone (1993) permitieron aislar los tres factores siguientes y por este orden: (1) miedo a la evaluación negativa (FNE), (2) evitación/distress social referido a situaciones especificas nuevas con los iguales (Social Avoidance and Distress-Specific to New Peers, SADN), y (3) evitación/distress social sobre situaciones generalizadas (Social Avoidance and Distress-General, SAD-G). Se observa, por tanto, que, aunque se mantiene la dimensión FNE, la antigua dimensión SAD deriva en las dos nuevas subescalas SAD-N y SAD-G. La Greca y Stone (1993) han referido evidencia factorial confirmatoria sobre la consistencia de esta estructura tridimensional, así como también datos empíricos sobre su fiabilidad y validez.

La escala SASC-R es breve, fácil de administrar y parece poseer buenas propiedades psicométricas. Por otra parte, es uno de los escasos instrumentos de autoinforme destinados específicamente a la evaluación de la ansiedad social en poblaciones de niños y adolescentes. Además, permite evaluar varias dimensiones relevantes para el estudio de la ansiedad $/ \mathrm{miedo}$ social, tanto a nivel clínico como no clínico.

La finalidad de esta investigación ha consistido en estudiar las dimensiones factoriales de la versión española de la SASC-R y SASC (Sandín, 1997) utilizando para ello una muestra de niños y adolescentes no clínicos. Puesto que, aparte de los trabajos llevados a cabo en USA, estas escalas han sido estudiadas con muestras de otros países, mediante la presente investigación pretendemos aportar validez transcultural sobre la estructura de la SASC-R y SASC y, por ende, validez transcultural sobre la estructura de la ansiedad social en población infantojuvenil. Las dimensiones de la escala se han investigado mediante la aplicación de técnicas de análisis factorial de componentes principales y rotaciones varimax y oblicua. De acuerdo con los datos referidos por los autores de la versión original de las escalas (La Greca et al., 1988; La Greca y Stone, 1993), y basándonos en las mencionadas escalas, nuestra primera hipóteis establece que la estructura de la ansiedad social es de naturaleza multidimensional. Según nuestra segunda hipóteis, la factorización de la versión española de la SASC-R debería dar lugar a una estructura tridimensional, consistente con las dimensiones FNE, SAD-N y SAD-G. Finalmente, mediante la tercera hipótesis, la factorización de la SASC debería dar como resultado dos factores representativos de las dimensiones FNE y SAD, respectivamente. 


\section{MÉTODO}

\section{Participantes}

Empleamos una muestra de 327 participantes normales (no clínicos ni con retraso mental), la cual estuvo constituida por 195 niños (media de edad $=12,53$, $\mathrm{DT}=2,14)$ y 133 niñas (media de edad $=$ $11,77, \mathrm{DT}=2,02$ ), con edades comprendidas entre 7 y 16 años. No existían diferencias significativas en edad según el sexo. Todos los participantes poseían un nivel socioeconómico medio. Los participantes asistían regularmente a colegios de enseñanza primaria y secundaria, y fueron seleccionados al azar en una zona urbana de la población escolar de la comunidad de Madrid (Boadilla del Monte).

\section{Procedimiento e instrumentos de medida}

Los sujetos fueron evaluados en su propio centro de estudios, al cual asistían con regularidad durante el curso académico. De forma colectiva se les aplicaron las escalas SASC-R y SASC, junto a otros cuestionarios no relevantes para el presente artículo. Las medidas se aplicaron de forma colectiva. Antes de su aplicación, el evaluador (tercer autor) leyó y explicó detenidamente las instrucciones de cada cuestionario. Así mismo, durante la cumplimentación de las pruebas, el evaluador permaneció en el aula para asistir a los niños/adolescentes y atender cualquier duda relacionada con la contestación a los cuestionarios. Aunque los cuestionarios se aplicaron de forma colectiva, el número máximo por grupo fue de 20 niños/adolescentes. A continuación detallamos brevemente ambos instrumentos de evaluación utilizados.

Escala de Ansiedad Social para NiñosRevisada [Social Anxiety Scale for Children-Revised] (SASC-R; La Greca y Sto- ne, 1993). Se aplicó la versión española de Sandín y Chorot (véase Sandín, 1997) ${ }^{1}$. La escala consta de 18 items, de los cuales, de acuerdo con los datos prelimiares de La Greca y Stone (1993) con población norteamerica, ocho items pertenecen a la dimensión FNE (items 2, 6 , $7,9,13,4,11$ y 14), seis a la dimensión SAD-N (items $1,3,5,8,10$ y 16), y cuatro a la dimensión SAD-G (items 12, 15, 17 y 18). En su cumplimentación, el niño/adolescente debe contestar de acuerdo a tres alternativas de respuesta para cada item, esto es, "Nunca» (1), "A veces» (2) y «Muchas veces» (3), según crea que el contenido de cada item sea más o menos frecuente en él/ella. En su versión original la SASC-R puede ser cumplimentada, bien según este criterio, o bien según una graduación de cinco niveles. Sin embargo, hemos utilizado la estructura más simple (i.e, de tres niveles) ya que esta modalidad suele ser más conveniente para su aplicación en niños.

Escala de Ansiedad Social para Niños [Social Anxiety Scale for Children] (SASC; La Greca et al., 1988; La Greca y Stone, 1993). Se aplicó la versión española de Sandín y Chorot (véase Sandín, 1997) de la SASC. La escala consta solamente de 10 items, y puede ser utilizada, en estudios que requieran menos tiempo de aplicación, para obtener una medida rápida sobre el nivel de ansiedad social. La SASC es la versión antigua de la escala, es más breve que la SASC-R, y se ha sugerido que su estructura está constituida por las dos dimensiones siguientes (en sus denominaciones se asignará una $\mathrm{O}$ [versión original] para no confundirlas con las versiones de la SASC-R): (a) FNEO (items 1, 2, 7, 9, 11 y 13 de la SASC-R), y (b) SAD-O (items 3, 5, 10 y 12 de la SASC-R).

\footnotetext{
${ }^{1}$ La versión española de la SASC-R puede encontrarla el lector en: B. Sandín, Ansiedad, miedos y fobias en niños y adolescentes, Madrid, Dykinson, 1997.
} 


\section{RESULTADOS}

\section{Estrategia de análisis factorial}

Con objeto de analizar la estructura factorial de la SASC-R y SASC se efectuaron análisis factoriales de componentes principales. Para poder obtener la máxima independencia entre los factores, e incrementar la interpretabilidad de la solución factorial rotada, así como también para comparar con los estudios norteamericanos sobre la escala, se aplicó rotación ortogonal (varimax). También aplicamos rotación oblicua (oblimín) debido a que: (1) asumimos que las dimensiones que subyacen a la escala correlacionan entre sí; y (2) debido a esta supuesta corrrelación, era necesario contrastar su estructura oblicua con la obtenida mediante rotación ortogonal.

Para la extracción del número de factores se tuvieron en cuenta criterios teóricos y empíricos. En primer lugar se consideró la estructura factorial sugerida previamente para las escalas SASC-R (tres factores) y SASC (dos factores), respectivamene, por $\mathrm{La}$ Greca y sus colaboradors (La Greca y Stone, 1993; La Greca et al., 1988). Según estos autores, la mejor estructura de la SASC-R consiste en tres factores, y la mejor estructura de la SASC consiste en dos factores. No obstante, aparte de estos criterios teóricos, para probar la posible relevancia de otras estructuras factoriales (p.ej., uno o dos factores), también empleamos los siguientes criterios empíricos de estimación: (1) criterio de Kaiser (1961), (2) criterios de extracción de Thurnstone (1947), (3) test de scree (Cattell, 1966), y (4) interpretabilidad de las estructuras resultantes (Gorsuch, 1983) que implica examinar soluciones con diferentes criterios de extracción. El criterio de Kaiser (i.e., consideración de los factores con autovalores por encima de la unidad), no obstante, en principio únicamente se tuvo en cuenta de modo orientativo, ya que, de tomarlo como exclusivo, puede derivar en un excesivo número de factores).

\section{Estructura factorial de la SASC-R}

Tras ser sometidos a análisis de componentes principales los 18 elementos de la SASC-R, se obtuvieron tres factores con valores eigen (autovalores) superiores a la unidad. La aplicación de los criterios de extracción indicados arriba sugerían una estructura de tres factores como mejor representante de la estructura simple, correspondiendo estos factores a las dimensiones FNE, SAD-G y SAD-N, respectivamente. Los tres factores explicaban el 52,5\% de la varianza total. En la Tabla 1 se muestran las saturaciones factoriales rotadas (varimax), los valores eigen, la varianza explicada y la comunalidad relativos a los tres factores.

El primer factor, que explica el $37,4 \%$ de la varianza total, está constituido por los elementos referidos a la dimensión de miedo a la evaluación negativa (FNE). Como puede observarse en la mencionada tabla, los items $6,7,9$ y 11 obtienen saturaciones en este factor por encima de 0,70 . Puede apreciarse, así mismo, que el resto de los elementos de esta dimensión alcanzan saturaciones elevadas en el factor (i.e., saturaciones $Æ 0,50$ ). Algunos items de la dimensión FNA, no obstante, presentan también pesos relevantes en el segundo factor; específicamente, los items 2, 4, 13 y 14 saturan por encima de 0,35 en el segundo factor. Estas saturaciones, sin embargo, son todas inferiores a las que se evidencian en el primer factor.

El segundo factor explica el 8,1\% de la varianza total y parece representar la dimensión de evitación y distress relativa a situaciones sociales generalizadas (SAD-G). Como puede apreciarse en la Tabla 1, los items referidos por La Greca y Stone (1993) como constitutivos de esta dimensión (items 12, 15, 17 y 18) tienden 
Tabla 1. Estructura factorial de la SASC-R; pesos factoriales tras rotación ortogonal (varimax) $(\mathrm{N}=327)$

\begin{tabular}{|c|c|c|c|c|}
\hline Item $S A S C-R$ & $\begin{array}{c}\text { Factor } 1 \\
(F N E)\end{array}$ & $\begin{array}{l}\text { Factor } 2 \\
(S A D-G)\end{array}$ & $\begin{array}{l}\text { Factor } 3 \\
(S A D-N)\end{array}$ & Comunalidad \\
\hline \multicolumn{5}{|l|}{ FNE } \\
\hline 2. Que me tomen el pelo & 0,60 & $\mathbf{0 , 4 1}$ & 0,28 & 0,38 \\
\hline 4. Que hablen de mi a mis espaladas & $\mathbf{0 , 5 0}$ & 0,35 & 0,08 & 0,46 \\
\hline 6. Me preocupa lo que piensen de mí & 0,75 & 0,11 & 0,28 & 0,65 \\
\hline 7. Caer mal a otros chicos & $\mathbf{0 , 7 6}$ & 0,13 & 0,25 & 0,66 \\
\hline 9. Que hablen de mí & 0,74 & 0,24 & 0,28 & 0,69 \\
\hline 11. No gustar a otros chicos & 0,74 & 0,06 & 0,23 & 0,66 \\
\hline 13. Que se burlen de mí & 0,60 & $\mathbf{0 , 4 3}$ & 0,01 & 0,55 \\
\hline 14. Discutir con otros chicos & $\mathbf{0 , 5 0}$ & 0,45 & 0,08 & 0,47 \\
\hline \multicolumn{5}{|l|}{ SAD-G } \\
\hline 12. Estar callado ante otros chicos & 0,12 & $\mathbf{0 , 5 6}$ & 0,38 & 0,56 \\
\hline 15. Miedo a que rehusen una invitación & 0,23 & $\mathbf{0 , 7 1}$ & 0,00 & 0,56 \\
\hline 17. Timidez ante chicos conocidos & 0,10 & $\mathbf{0 , 5 9}$ & 0,25 & 0,42 \\
\hline 18. Pedir que jueguen conmigo & 0,13 & 0,63 & 0,23 & 0,47 \\
\hline \multicolumn{5}{|l|}{ SAD-N } \\
\hline 1. Hacer cosas nuevas ante otros niños & 0,34 & 0,41 & 0,30 & 0,37 \\
\hline 3. Timidez ante chicos desconocidos & 0,16 & 0,14 & 0,73 & 0,57 \\
\hline 5. Evitar chicos no conocidos & 0,06 & 0,25 & 0,45 & 0,47 \\
\hline 8. Nerviosismo ante chicos poco conocidos & 0,30 & 0,20 & $\mathbf{0 , 7 2}$ & 0,64 \\
\hline 10. Nervisismo ante nuevos chicos & 0,31 & 0,21 & 0,75 & 0,70 \\
\hline 16. Nerviosismo ante ciertos chicos & 0,33 & 0,39 & 0,35 & 0,38 \\
\hline Valor eigen & 6,73 & 1,46 & 1,27 & \\
\hline Porcentaje de varianza & $37,40 \%$ & $8,10 \%$ & $7,00 \%$ & \\
\hline
\end{tabular}

Nota: Los enunciados de los items aparecen de forma abreviada. Los pesos factoriales elevados $(\geq 0,40)$ se indican en negrita. Los items están separados por subescalas (FNE, SAD-G y SAD-N) según la agrupación original de La Greca y Stone (1993). FNE = fear of negative evaluación, SAD-G = social avoidance and distress-general, SAD$\mathrm{N}=$ social avoidance and distress-specific to new peers.

a saturar de forma selectiva en este segundo factor (sólo el item 12 posee un peso elevado en otro factor -en este caso en el tercer factor). En el Factor 2, sin embargo, otros items del cuestionario no pertenecientes a la dimensión SAD-G presentan pesos superiores o cercanos a 0,40 (items $2,4,13,14,1$ y 16). Los dos últimos items presentan el problema de poseer saturaciones más elevadas en este factor que en el tercer factor (factor relacionado con la dimensión SAD-N).

El tercer factor (explica el $7,0 \%$ de la varianza total) parece representar la dimensión de evitación y distress social relativa a situaciones nuevas (SAD-N). Entre los seis items propuestos por La Greca y Stone (1993) para la dimensión SAD-N, tres (items 3, 8 y 10) alcanzan valores sobresalientes $(\geq 0,70)$. Los items 1 y 16, aparte de obtener pesos superiores en el Factor 2 que en el tercer factor, saturan $(\geq 0,30)$ en los tres factores.

\section{Estructura factorial de la SASC}

La SASC (forma antigua de la escala) puede resultar de utilidad cuando se desea obtener una indicación rápida sobre la ansiedad social (consta sólo de 10 items). El análisis factorial dio como resultado dos factores con valores eigen superiores a la unidad. Los dos factores explicaban el $53 \%$ de la varianza total. Los criterios de estimación sugerían que la solución factorial de dos factores resultaba ser la más adecuada. En la Tabla 2 
indicamos los pesos factoriales rotados (varimax), los valores eigen, la varianza explicada y la comunalidad para ambos factores.

El primer factor corresponde a la dimensión FNE-O. Entre los cinco items que conforman esta dimensión, sólo el item 13 satura de forma destacada $(\geq$ 0,40 ) en el segundo factor.

El segundo factor está, en general, configurado por los items de la dimensión SAD-O. Unicamente el item 1 presenta una saturación importante $(\geq 0,40)$ en el otro factor (primer factor). En este caso, se da la circunstancia de que dicha saturación es incluso mayor que la exhibida en el segundo factor).

\section{Resultados con el procedimiento de} rotación oblicua

Ambas subescalas (SASC-R y SASC) fueron también sometidas al procedimiento de rotación oblicua (oblimín). Los resultados obtenidos, tras haber extraído tres (SASC-R) y dos (SASC) factores, respectivamente, fueron similares a los indi- cados para el procedimiento de rotación ortogonal, sin excepciones. Los mismos items, y en medidas similares, que presentaban saturaciones en los factores no congruentes tras rotación ortogonal (véanse las Tablas 1 y 2) lo hacían tras rotación oblicua. Curiosamente las correlaciones entre los factores de la SASC-R en la rotación oblicua no fueron excesivamente elevados: $r=0,43$ (entre $F 1$ y F2); $r=0,23$ (entre F1 y F3); y $r=0,27$ (entre F2 y F3). Esto sugiere la posibilidad de entender estos factores como dimensiones relativamente independientes de la ansiedad social. La correlación entre los dos factores de la escala SASC fue ligeramente más elevada $(r=0,48)$.

\section{DISCUSIÓN}

Mediante el presente estudio presentamos la versión española del cuestionario de autoinforme SASC-R de La Greca y Stone (1993) (así como también de la versión inicial y más breve SASC), el cual fue diseñado para la evaluación de la ansiedad social en niños y adolescentes.

Tabla 2. Estructura factorial de la SASC; pesos factoriales tras rotación ortogonal (varimax) $(\mathrm{N}=327)$

\begin{tabular}{lccc}
\hline Item SASC & $\begin{array}{l}\text { Factor 1 } \\
\text { (FNE-O) }\end{array}$ & $\begin{array}{c}\text { Factor 2 } \\
\text { (SAD-O) }\end{array}$ & Comunalidad \\
\hline FNE-O & & & \\
2. Que me tomen el pelo & $\mathbf{0 , 6 4}$ & 0,08 & 0,42 \\
7. Caer mal a otros chicos & $\mathbf{0 , 8 3}$ & 0,13 & 0,70 \\
9. Que hablen de mí & $\mathbf{0 , 7 6}$ & 0,30 & 0,67 \\
11. No gustar a otros chicos & $\mathbf{0 , 7 9}$ & 0,17 & 0,66 \\
13. Que se burlen de mi & $\mathbf{0 , 4 8}$ & $\mathbf{0 , 4 2}$ & \\
SAD-O & & & \\
1. Hacer cosas nuevas ante otros & $\mathbf{0 , 4 7}$ & $\mathbf{0 , 4 3}$ & $\mathbf{0 , 4 0}$ \\
3. Timidez ante chicos desconocidos & 0,29 & $\mathbf{0 , 5 9}$ & $\mathbf{0 , 4 4}$ \\
5. Evitar chicos no conocidos & 0,09 & $\mathbf{0 , 7 2}$ & $\mathbf{0 , 5 3}$ \\
10. Nervisismo ante nuevos chicos & 0,36 & $\mathbf{0 , 5 4}$ & $\mathbf{0 , 5 1}$ \\
12. Estar callado ante otros chicos & 0,24 & $\mathbf{0 , 7 3}$ & $\mathbf{0 , 5 9}$ \\
Valor eigen & & & \\
Porcentaje de varianza & 4,17 & $\mathbf{1 , 1 4}$ & \\
\hline
\end{tabular}

Nota: Los enunciados de los items aparecen de forma abreviada. Los pesos factoriales elevados $(\geq 0,40)$ se indican en negrita. Los items están separados por subescalas según la agrupación de La Greca y Stone (1993). FNE-O = fear of negative evaluation (versión original), SAD-O = social avoidance and distress (versión original). 
Los resultados de nuestra investigación proporcionan apoyo empírico a la estructura factorial del cuestionario (i.e., confirmación de sus dimensiones o subescalas) y, en último término, apoyo a la estructura multidimensional de la ansiedad social.

En lo que concierne a la estructura factorial de la SASC-R, nuestros datos sugieren que la versión española del cuestionario, al igual que la versión inglesa, posee tres factores que representan las dimensiones de miedo a la evaluación negativa (FNE), evitación/distress social generalizado (SAD-G), y evitación/distress social ante situaciones con nuevos niños (SAD-N). Estos resultados, en términos generales, corroboran los datos sobre las dimensiones del cuestionario presentados por La Greca y Stone (1993), quienes definieron conceptual y empíricamente estas tres dimensiones (o subescalas) como constitutivas de la estructura de la SASC-R. Existen, sin embargo, algunas diferencias, que comentamos a continuación, entre los resultados referidos por estos autores y los obtenidos por nosotros en el presente estudio.

Tal vez el aspecto más destacable es que los tres factores que conforman la SASC-R no están tan claramente definidos en nuestros datos como los indicados por La Greca y Stone (1993), ya que, como hemos visto en la sección sobre los resultados, algunos items de la escala tienden a saturar en otro factor, aparte de hacerlo en el factor relevante. Este es el caso de los items 2, 13 y 14 (FNE), 12 (SAD-G), y 1 y 16 (SAD-N). Los items 2, 13 y 14, además de saturar en el Factor 1 (FNE) poseen pesos elevados en el Factor 2 (SAD-G), lo cual podría indicar que tales items no son medidas puras del miedo a la evaluación negativa, sino que además forman parte del constructo de evitación/distress social generalizado. Estos tres items, a tenor de su contenido, no parece que se refieran específicamen- te al miedo a la evaluación negativa, sino a un fenómeno más general de ansiedad social que incluye, entre otros componentes, el propio miedo a la evaluación negativa. Resulta interesante resaltar, por otra parte, que en el estudio de La Greca y Stone (1993) estos mismos items también presentaban saturaciones altas en los factores de SAD.

El item 12, aunque pertenece a la dimensión SAD-G, satura así mismo de forma significativa en la dimensión SAD-N, fenómeno que también se evidencia en el análisis factorial de la versión original de la escala (La Greca y Stone, 1993). Los items 1 y 16 presentan el problema de saturar en los tres factores y no presentar saturaciones muy sobresalientes en ninguno de ellos. El item 1 había sido considerado inicialmente por La Greca como constitutivo de la dimensión FNE. Según nuestros análisis, parece referirse a la ansiedad social en general, más que a dimensiones específicas. Por otra parte, a nuestro juicio, la descripción de este item se diferencia del resto de los items de la dimensión SAD-N porque se refiere a situaciones nuevas, y no a compañeros nuevos o poco conocidos.

El item 16, finalmente, presenta saturaciones muy semejantes en los tres factores. Esto podría deberse a que el enunciado de dicho item presenta cierta ambigüedad, lo cual hace que, conceptualmente, podría asignarse a cualquiera de las tres dimensiones de la escala. De hecho, si nos atenemos a su enunciado, el sentirse nervioso cuando se está cerca de ciertos chicos puede asociarse al miedo ante la evaluación negativa de los otros (chicos/as), o bien al distress generado por la presencia de muchos otros chicos («ciertos» niños no indica que éstos implique necesariamente situaciones «nuevas»). El item, por tanto, resulta escasamente discriminativo con respecto a la diferenciación de las dimensiones de 
la SASC-R y tal vez debería ser eliminado o reformulado.

El análisis factorial de la SASC apoya la hipótesis sobre las dos dimensiones (FNE-O y SAD-O) (véase la Tabla 2) sugeridas por el grupo de La Greca. Las posibles implicaciones de las saturaciones en el factor no congruente observadas para los items 13 y 1 son equivalentes a las que hemos indicado a propósito de analizar la estructura factorial de la SASC-R.

Es importante resaltar que las correlaciones entre las dimensiones (factores) de las escalas (especialmente de la SASC-R) han resultado ser moderadamente bajas. Este es un hecho importante pues, aparte de indicar que la estructura de la ansiedad social (al menos en niños y adolescentes) es claramente de naturaleza multifactorial, sugiere la adecuación (y posiblemente conveniencia) de emplear, además de las puntuaciones totales de las escalas, las dimensiones (i.e., subescalas) de las mismas tanto para la investigación como para la evaluación clínica de la ansiedad social.

A modo de conclusión, en el presente artículo presentamos la versión española del cuestionario SASC-R (así como también la anterior versión SASC) y los primeros datos empíricos sobre su estructura factorial. Que nosotros sepamos, esta es la primera vez que la SASC-R ha sido validada con población española. Puesto que éste es uno de los pocos cuestionarios que existen actualmente para evaluar la ansiedad social en niños y adolescentes, el cuestionario podría resultar de gran interés y utilidad para la investigación y tratamiento de los problemas relacionados con la ansiedad social en es tipo de población.

\section{REFERENCIAS BIBLIOGRÁFICAS}

American Psychiatric Association (APA) (1994). Diagnostic and statistical manual of mental disorders (4th ed.). Whasington, DC: Autor.
Botella, C., y Gallardo, M. (1999). La fobia social. Madrid: UNED-FUE.

Cattell, R.B. (1966). The scree test for the number of factors. Multivariate Behavioral Rerearch, 3, 245-276.

Echeburúa, E. (1993). Fobia social. Barcelona: Martínez Roca.

Gorsuch, R. (1983). Factor analysis. Hillsdale, NJ: Erlbaum.

Heimberg, R.G., Holt, C.S., Schneier, F.R., Spitzer, R.L., y Liebowitz, M.L. (1993). The issue of subtypes in the diagnosis of social phobia. Journal of Anxiety Disorders, 7, 249-269.

La Greca, A.M., Dandes, S.K., Wick, P., Shaw, K., y Stone W.L. (1988). Development of the Social Anxiety Scale for Children: Reliability and concurrent validity. Journal of Clinical Child Psychology, 17, 84-91.

La Greca, A.M., y Stone, W.L. (1993). Social Anxiety Scale for Children-Revised: Factor structure and concurrent validity. Journal of Clinical Child Psychology, 22, 17-27.

Leary, M.R. (1983). A brief version of the Fear of Negative Evaluation Scale. Personality and Social Psychology Bulletin, 9, 371-375.

Méndez, F.X. (1999). Miedos y temores en la infancia. Madrid: Pirámide.

Pelechano, V. (1983). Miedos infantiles y terapia familiar natural. Valencia: Alfaplús.

Sandín, B. (1997). Ansiedad, miedos y fobias en niños y adolescentes. Madrid: Dykinson.

Sandín, B., y Chorot, P. (1995). Síndromes clínicos de la ansiedad. En A. Belloch, B. Sandín y F. Ramos (Eds.), Manual de psicopatología, Vol. 2 (pp. 81-112). Madrid: McGraw-Hill.

Sandín, B., y Chorot, P. (1998). Dimensiones de los miedos en los niños: Estructura factorial del FSSC-R. Psiquis, 19, 23-32.

Sandín, B., Chorot, P., Valiente, R.M., y Santed, M.A. (1998). Frecuencia e intensidad de los miedos en los niños: Datos normativos. Revista de Psicopatología y Psicología Clínica, 3, 15-25.

Thurstone, L.L. (1947). Multiple factor analysis. Chicago: University of Chicago Press.

Watson, D. y Friend, R. (1969). Measurement of social-evaluative anxiety. Journal of Consulting and Clinical Psychology, 33, 448-457. 